\title{
Represión de actos lesivos homogéneos y la tutela jurisdiccional efectiva del personal docente que laboró bajo el régimen de las leyes 24029 y 25212, en el Perú
}

\author{
Repression of homogeneous harmful acts and the effective \\ jurisdictional protection of teaching staff who worked under \\ the regime of laws 24029 and 25212, of Perú
}

\author{
Ericson Delgado Otazu ${ }^{[*]}$
}

\begin{abstract}
Resumen: todo Estado está comprometido a garantizar a todo sujeto de derecho el acceso a la justicia, suministrando recursos judiciales efectivos a quienes se sientan agraviados; para ello, cada Estado debe optar con las medidas correspondientes para eliminar todo impedimento que obstaculice el pleno ejercicio de tal derecho; en el Perú contamos con la institución de la represión de actos lesivos homogéneos, regulado de manera genérica en el art. 60 del Código Procesal Constitucional; sin embargo, podría ser explotado y empleado con resultados muy favorables para garantizar una tutela jurisdiccional realmente efectiva, en determinados casos por ejemplo, los que involucran el reconocimiento de beneficios laborales del sector educación.
\end{abstract}

Palabras claves: represión, actos, homogéneos, tutela, jurisdiccional.

Abstract: every state is committed to guaranteeing to all legal subjects access to justice by providing effective judicial remedies to those who feel aggrieved; for this, each state must opt for the corresponding measures to eliminate any impediment that hinders the full exercise of such right; in Perú we have the institution of the repression of homogeneous harmful acts, regulated in a generic way in art. 60 of the Constitutional Procedural Code; however, it could be exploited and used with very favorable results to guarantee a really effective jurisdictional protection, in certain cases, for example, those that involve the recognition of labor benefits in the education sector.

Key words: repression, acts, homogeneous, protection, jurisdictional.

[*] Abogado por la Universidad Nacional San Antonio Abad del Cusco, Doctor en Derecho por la Universidad Andina del Cusco, Docente de la Escuela Profesional de Derecho de la UNSAAC y la UAC. ericson.delgado@ unsaac.edu.pe. 


\section{INTRODUCCIÓN}

El derecho al acceso a la justicia se encuentra consagrado en el artículo 25 de la Convención Americana sobre Derechos Humanos $(\mathrm{CADH})$, bajo la designación de «Protección Judicial», en el Derecho Comparado y en nuestra legislación a este derecho se le denomina Tutela Jurisdiccional Efectiva. Para el jurista español Jesús González Pérez la Tutela Jurisdiccional Efectiva «es el derecho de toda persona a que se le haga justicia; a que cuando pretenda algo de otra, esta pretensión sea atendida por un órgano jurisdiccional, a través de un proceso con garantías mínimas» (Gonzales, 1985, p. 27).

En el plano nacional contamos que este derecho se encuentra regulado en varios instrumentos normativos tales como el inciso 3 del artículo 139 de nuestra Constitución Política, el artículo 4 del Código Procesal Constitucional, el artículo I del Título Preliminar del Código Procesal Civil y el artículo 7 de la Ley Orgánica del Poder Judicial.

En esta línea nuestro Código Procesal Constitucional reconoce en su artículo 60 la institución procesal de Represión de Actos Homogéneos Lesivos, del cual el Tribunal Constitucional se pronunció estableciendo lo siguiente:

La represión de actos lesivos homogéneos es un mecanismo de protección judicial de derechos fundamentales frente a actos que exhiben características similares a aquellos que en una sentencia previa han sido considerados contrarios a tales derechos. En este sentido lo resuelto en un proceso constitucional de tutela de derechos fundamentales no agota sus efectos con el cumplimiento de lo dispuesto en sentencia respectiva, sino que se extiende hacia el futuro en la perspectiva de garantizar que no se vuelva a cometer una afectación similar del mismo derecho. (Exp. N. ${ }^{\circ}$ 01345-2013-PA/TC, 2013)

Esta institución insta de manera urgente, los procesos constitucionales, los cuales, si el tribunal constitucional los resuelve de manera favorable en una sentencia, servirá como antecedentes frente a otros procesos de similares características.

En el presente caso, hacemos referencia al personal docente del sector educación quienes laboraron durante la vigencia de la Ley del Profesorado N. ${ }^{\circ} 24029$ y su modificatoria Ley N. ${ }^{\circ} 25212$ específicamente desde el 21 de mayo de 1990 al 25 de noviembre de 2012 (fecha de derogatoria de la Ley del Profesorado).

En la Ley del profesorado se reconoce el derecho de los docentes a percibir una bonificación especial por preparación de clases y una bonificación adicional por desempeño de cargo y elaboración de documentos de gestión, el cual viene a ser el $30 \%$ y $5 \%$ respectivamente del integro de la remuneración; sin embargo; en el mes de marzo del año 1991, se emite el Decreto Supremo 051-91- PCM, disponiendo que:

A partir del 01 de febrero de 1991, se deje sin efecto, transitoriamente, sin excepción, las disposiciones legales y administrativas que establezcan remuneraciones mensuales tomando como referencia el ingreso total y otros beneficios de carácter mensual que perciban los Senadores y Diputados. (Art. 2)

Es así que, el Artículo $10^{\circ}$ del referido Decreto Supremo 051-91- PCM, regula lo siguiente: «Precisase que lo dispuesto en el Artículo $48^{\circ}$ de la Ley del Profesorado N. ${ }^{\circ} 24029$, modificada por Ley $\mathrm{N}^{\circ}$ 25212, se aplica sobre la Remuneración Total Permanente establecida en el presente Decreto Supremo».

Desde la entrada en vigencia del Decreto Supremo 051-91- PCM, hasta la derogatoria de la Ley del Profesorado, 25 de noviembre de 2012, y en caso de los docentes cesantes, antes de dicha fecha, bajo el régimen del Decreto Legislativo N. ${ }^{\circ} 20530$, la administración pública, específicamente el Ministerio de Educación, viene efectuando el pago de la bonificación especial por preparación de clases, desempeño de cargo y elaboración de documentos de gestión, tomando como referencia de 
cálculo la bonificación total permanente más no la remuneración total íntegra, y para diferenciar ambas remuneraciones, basta remitirnos al Art. $8^{\circ}$ del mismo Decreto Supremo 051-91PCM, definiéndolo de la forma siguiente:

Artículo $8^{\circ}$.- Para efectos remunerativos se considera:

a. Remuneración total permanente.- Aquella cuya percepción es regular en su monto, permanente en el tiempo y se otorga con carácter general para todos los funcionarios, directivos y servidores de la Administración Pública; y está constituida por la Remuneración Principal, Bonificación Personal, Bonificación Familiar, Remuneración Transitoria para Homologación y la Bonificación por Refrigerio y Movilidad.

b. Remuneración total.- Es aquella que está constituida por la Remuneración Total Permanente y los conceptos remunerativos adicionales otorgados por Ley expresa, los mismos que se dan por el desempeño de cargos que implican exigencias y/o condiciones distintas al común.

Siendo un poco más explícito, aplicando el criterio de la remuneración total permanente, la bonificación especial dispuesta en el Art. $48^{\circ}$ de la Ley del Profesorado, equivalente al $30 \%$ de dicha remuneración asciende a un promedio de S/. 20.00, por el contrario, aplicando el 30\% a la remuneración total, la bonificación especial sería aproximadamente de S/. 300 , en el entendido de que la remuneración total de un profesor al 2012 ascendía aproximadamente a S/. 1000.00.

Es por ello, que los docentes del sector educación, advertidos de tal discrepancia e injusticia, acuden al órgano jurisdiccional en busca de tutela efectiva, pretendiendo el reconocimiento, reintegro y pago de lo dispuesto en el Art. $48^{\circ}$ de la Ley del Profesorado, vale decir el re-cálculo de la bonificación especial en base a la remuneración total, que incluye el crédito devengado desde mayo de 1990, la nivelación de la pensión en caso de docentes cesantes y para todos los casos el pago de los intereses legales. Obteniendo en el común de los casos sentencia favorable, dado que los magistrados a cargo de dichos procesos ponderan la aplicación del principio de especialidad y el de jerarquía de normas; el primero, en razón de la preferencia de la norma especial al momento de su aplicación frente a una norma de carácter general, pues la Ley del Profesorado, regula el supuesto de hecho específico, del pago de la bonificación especial sobre la base de la remuneración total, mientras que el Decreto Supremo 051-91-PCM regula la determinación de las bonificaciones de manera genérica sobre la base de la remuneración total permanente; y el segundo, en razón de lo dispuesto por el Artículo $51^{\circ}$ de la Constitución Política del Estado, respecto a la prevalencia de la norma constitucional sobre toda norma legal y de la ley, sobre las normas de inferior jerarquía, vale decir Ley del Profesorado es jerárquicamente superior que el Decreto Supremo 051-91-PCM; por tanto, debe primar y aplicar la primera.

Es cierto que administrativamente el personal docente no puede reivindicar su derecho al pago de la bonificación especial sobre la base del $30 \%$ de la remuneración total, principalmente, por las disposiciones presupuestales y los lineamientos emitidos por el Ministerio de Educación; motivo por el cual, obligatoriamente debe recurrir a la instancia judicial y ya cuando cuenta con una sentencia judicial con autoridad de cosa juzgada, puede exigir el pago de la citada bonificación que por derecho le corresponde.

Y para llegar a ese momento, el docente debe atravesar varios obstáculos, tanto en la vía administrativa como judicial, tales como la demora en la tramitación, lentitud de la administración pública, excesiva carga procesal, carencia de economía para afrontar los gastos del proceso, principalmente honorarios del abogado patrocinante, demora en la ejecución de sentencia, entre otros, los mismos que constituyen una limitante para no iniciar el pro- 
ceso judicial y lamentablemente quedarse resignados a no gozar del pago de dicha deuda.

Este derecho reconocido, pero no amparado por la Administración Pública ha dado origen a un conjunto de procesos judiciales a nivel nacional, generando una excesiva carga procesal; sin embargo, pese a existir la institución de Actos Lesivos Homogéneos, y habiendo un precedente de su uso en los casos de sepelio y de luto, en la práctica los docentes demandantes no hacen uso de este instrumento.

\section{REPRESIÓN DE ACTOS LESIVOS HOMO- GÉNEOS}

El Tribunal Constitucional (TC) precisó con meridiana claridad los alcances de la institución denominada represión de actos lesivos homogéneos contemplada en el artículo 60 ํ del Código Procesal Constitucional, en la perspectiva de evaluar su aplicación en caso concreto, para ello decidió declarar fundado el recurso de queja por denegatoria del recurso de agravio constitucional formulado por un ciudadano, que a pesar de haber obtenido respuesta favorable en la segunda instancia del Poder Judicial, insistió en su pretensión. (Rioja, 2012, p. 05)

El Tribunal Constitucional afirma que la represión de los actos lesivos homogéneos es un mecanismo de protección judicial de los derechos fundamentales, frente actos de la vulneración de los derechos, que se han considerado una sentencia previa, por consiguiente un proceso constitucional de tutela de derechos fundamentales agota sus efectos a efectuarse el cumplimiento de la disposición de la sentencia respectiva, sin extender el tiempo del proceso, esta perspectiva garantiza de que no vuelva a incurrir a una afectación similar del derecho la represión de actos lesivos homogéneos tiene su fundamento en la necesidad de asegurar la vinculatoriedad de la sentencia ejecutada, para evitar un nuevo proceso constitucional.

El Código Procesal Constitucional señala literalmente sobre esta institución que constitu- ye una institución procesal que consolida el carácter de urgencia de los procesos constitucionales, en tanto permite dos efectos definidos: de un lado, evita la interposición de un nuevo proceso constitucional frente a una determinada agresión de similares caracteres a aquella que ocurrió en un primer proceso, en cuya ejecución se produce una nueva agresión, y de otro lado, implica una pronta actuación de la justicia constitucional frente a agresiones que vulneren derechos fundamentales.

Determina los parámetros de una futura agresión que se asemeja a una ya habida para fijar una tutela de derechos para aquellos vulnerados, ahorrando tiempo en la resolución de esa controversia.

Esta institución garantiza que los procesos constitucionales sean resueltos de manera urgente, impidiendo la interposición de nuevas demandas referidos a hechos similares o afectaciones similares de derechos fundamentales; en tanto que, en el primer proceso ya se hizo el análisis de los hechos y se concluyó en la afectación del derecho fundamental, lo que corresponde en los nuevos casos es la adecuación de los hechos a lo ya resuelto, acogiéndose a los efectos de la sentencia a través de la represión de actos lesivos homogéneos.

Situación que generara una pronta actuación de justicia constitucional frente a agresiones que transgredan derechos fundamentales del individuo, evitando el inicio y conclusión de un nuevo proceso, pues los efectos de una sentencia de orden constitucional podrán ser aplicados a hechos idénticos que representa una agresión al mismo derecho fundamental.

Desde la dación del Código Procesal Constitucional en 2004, el Perú ha afianzado un interesante camino de defensa de los derechos fundamentales, fijando la consolidación de distintas instituciones procesales, propias y del Derecho Comparado, para un encaminamiento más presto contra las agresiones verticales y horizontales respecto a los derechos tutelados por nuestra Carta Fundamental. (Figueroa, 2013) 
La actual norma constitucional adjetiva, incorpora novedosos mecanismos de defensa de los derechos fundamentales, recogiendo diversas instituciones procesales, entre ellas la represión de actos lesivos homogéneos, figura poco difundida y con escasa información tanto en nuestro medio como en la doctrina jurisprudencial comparada, la misma que es útil para la protección de agresiones reiteradas de los mismos derechos fundamentales.

Para la tutela de los derechos vulnerados al personal docente que laboró durante la vigencia de la Ley de Profesorado, materia del presente análisis, sería muy útil la aplicación de los actos lesivos homogéneos, correspondiendo a la protección de estos derechos una eficacia vertical; pues, la agresión consistente en la omisión del pago de bonificaciones dispuestas por ley, proviene del Estado, a diferencia de la agresión horizontal que se da entre particulares.

Nuestro Código Procesal Constitucional es el primero en su género en Iberoamérica respecto a un país y en propiedad, ha afianzado a nuestra nación en un lugar expectante en la defensa de los derechos fundamentales, en tanto no solo hemos previsto la proyección de un instrumento procesal autónomo como nuestro Código sino que hemos establecido una doctrina jurisprudencial- obra del Tribunal Constitucional y del Poder Judicial- orientada a una defensa más efectiva de los derechos fundamentales, y por cierto, también en otro sentido necesariamente reguladora de las peticiones que no llegan a satisfacer un estándar de protección de un derecho fundamental. (Figueroa, 2013)

El Perú fue uno de los primeros países, en contar con una apropiada regulación en materia de defensa de los derechos fundamentales, a través del Código Procesal Constitucional, con una amplia doctrina jurisprudencial, que ayuda a la interpretación de y aplicación de sus institutos, como es el caso de la represión de actos lesivos; pues, pese a la escasa doctrina y jurisprudencia, existen sentencias emitida por el propia Tribunal Constitucional que orientan y reglamentan su aplicación, a diferencia de otros países que contando con dicha figura en su legislación, no la hacen uso. Es por ello que nuestro país destaca en Iberoamérica en una adecuada regulación de la defensa efectiva de los derechos fundamentales, contando con mecanismos eficaces para una segura y pronta protección de derechos, dentro de ellos, la represión de actos lesivos, el mismo que debería ser utilizado con mayor frecuencia, por lo que me permito proponer su aplicación en la reivindicación de los derechos vulnerados del personal docente.

La represión de actos homogéneos constituye una institución procesal que consolida el carácter de urgencia de los procesos constitucionales, en tanto permite dos efectos definidos: de un lado, evita la interposición de un nuevo proceso constitucional frente a una determinada agresión de similares caracteres a aquella que ocurrió en un primer proceso, en cuya ejecución se produce una nueva agresión, y de otro lado, implica una pronta actuación de la justicia constitucional frente a agresiones que vulneren derechos fundamentales. (Figueroa, 2013)

Los procesos constitucionales por su propia naturaleza son bastante céleres, no se admite actuaciones que entorpezcan ni dilaten la secuela del proceso, siendo la represión de actos homogéneos un instrumento que refleja en estricto este carácter, por ser un mecanismo de protección judicial de derechos fundamentales, basado en hechos ya reconocidos y discutidos previamente en una sentencia con rango constitucional y que sirven de sustento para resolver nuevas afectaciones producidas por los mismos hechos. Con la represión de actos lesivos homogéneos, se ratifica la naturaleza urgente de los procesos constitucionales impidiendo la generación de nuevos procesos por las mismas afectaciones a derechos fundamentales, logrando una pronta justicia. 
La figura de la represión de actos homogéneos constituye un aporte que expresa una de las vertientes del principio de elasticidad constitucional, así como del de autonomía procesal respecto a los procesos constitucionales, en manifiesta expresión de ese carácter de tutela urgente que parte de la propia. (Figueroa, 2013)

La elasticidad constitucional es un principio dirigido al juzgador, teniendo este la libertad de adecuar el proceso a las necesidades y exigencias de la justicia, propiamente a la urgencia que representa, pudiendo suprimir o recortar actos procesales para lograr los fines del proceso constitucional, vale decir garantizar la preponderancia de la Constitución Política del Estado y la efectiva vigencia de los derechos reconocidos por la norma constitucional. Siendo la represión de actos homogéneos un instrumento apropiado para efectivizar el principio de elasticidad y autonomía procesal en materia constitucional.

Es por esta razón que la autonomía constitucional le confiere al tribunal constitucional un grado de responsabilidad la cual le permite generar principios con pretensión de generalidad a través de la doctrina jurisprudencial.

Resulta a veces compleja la determinación de la urgencia o la fijación de los caracteres de un recurso sencillo y rápido, dado que muchas veces expresan conceptos semánticamente muy abiertos y de sintaxis ciertamente exigente, y sin embargo, la apuesta por una presta y pronta tutela de los derechos fundamentales conduce a que los legisladores, de inicio al configurar la norma, y los jueces, en su tarea de configuración jurisprudencial de los derechos fundamentales, busquen el afianzamiento de instituciones, como ésta de la represión de actos homogéneos, a fin de consolidar una política de tutela real y no nominal de los derechos protegidos por la Carta Fundamental. (Figueroa, 2013)
Ciertamente habrá de ser necesario configurar en el camino las exigencias procedimentales del caso, pues es necesario fijar a los demás intérpretes del sistema las reglas de juego a utilizar, en tanto y en cuanto es en propiedad el Tribunal Constitucional, el órgano supremo encargado de fijar los mecanismos de aplicación de este tipo de figuras, bajo reglas de predictibilidad a ser aplicadas idóneamente por los jueces constitucionales del Poder Judicial, logrando con la práctica consolidar el uso y aplicación de la represión de actos homogéneos, del mismo modo, generalizar la protección de los derechos fundamentales con el empleo de esta institución, consiguiendo enormes satisfacciones en la búsqueda de alcanzar justicia.

\subsection{Juez competente}

El juez que conoce la represión de actos lesivos homogéneos es el juez de ejecución, que como regla general es el juez que conoció en primera instancia la demanda que dio inicio al proceso constitucional.

Se precisa así la figura de un juez que defienda el derecho y que, al mismo tiempo, se vea precisado a denegar el mismo, según el caso concreto, siempre que ocurra que no se configuren las condiciones del caso para su goce. (Figueroa, 2013)

Un juez deberá actuar en el marco de la legalidad, lo que significaría que sea estricto respecto a los elementos exigidos para constituir un acto lesivo homogéneo, y en su discrecionalidad, podrá considerar que un determinado caso no tiene los componentes necesarios para delimitarlo como tal, es importante la labor del juez que conocerá la solicitud de represión de actos lesivos, pues debe confrontar los nuevos hechos atentatorios de derechos fundamentales con los ya analizados en el proceso constitucional principal, que sin duda puede resultar una labor no muy compleja por el previo estudio y conocimiento del primer proceso. 
La represión de actos homogéneos está establecida en el artículo $60^{\circ}$ del Código Procesal Constitucional (C.P. Const.), lo cual menciona que: si sobreviniera un acto sustancialmente homogéneo al declarado lesivo en un proceso de amparo, podrá ser denunciado por la parte interesada ante el juez de ejecución. Efectuado el reclamo, el Juez resolverá éste con previo traslado a la otra parte por el plazo de tres días. La resolución es apelable sin efecto suspensivo. La decisión que declara la homogeneidad amplía el ámbito de protección del amparo, incorporando y ordenando la represión del acto represivo sobreviviente. (Eto, 2016, p. 276)

En este caso la norma desprende que las declaraciones innovativas no son irrelevantes, en si la demarcación de un acto lesivo primigenio es indispensable para determinar con posterioridad la sentencia de un acto homogéneo o esencialmente idéntico. $Y$ al determinarse como tal, se seguirá un proceso positivamente diferenciado, que significa un procedimiento ágil y diligente que protegerá de mejor forma un derecho fundamental, pues el plazo que tiene el juez para decidir es menor a un proceso constitucional ordinario.

De esta manera podemos considerar que la represión de actos lesivos homogéneos es un instrumento de protección judicial que presentan actos similares considerados en una sentencia, los cuales fija alcances de una nueva tutela en relación al derecho transgredido teniendo como resultado un ahorro en el proceso. En este contexto el proceso constitucional que tutela de derechos fundamentales garantiza el cumplimiento de una sentencia, que no solo será útil para un caso en concreto, sino persistirá, extendiéndose hacia el futuro.

\subsection{Exigencias para la configuración de los ac- tos homogéneos}

Para la correcta aplicación de la represión de actos lesivos, es necesaria la concurrencia de algunas exigencias, dada la connotación de los derechos fundamentales protegidos.
«La determinación de actos homogéneos fija dos ámbitos previos necesariamente correlacionados por existir una decisión antecedente y un hecho a denominar consecuente que en suma se pretende contrastar con el acto matriz de vulneración del derecho fundamental concernido» (Figueroa, 2013)

Estas exigencias son las siguientes:

\section{a) Existencia de una sentencia ejecutoriada a favor del demandante en un proceso constitucional de tutela de derechos fun- damentales}

Este requerimiento sigue la pauta de un necesario pronunciamiento con sentencia realizable a partir de su propia condición de decisión ejecutoriada. Es en base a la consecuencia fundamental que fije esa decisión previa que podrá determinarse si un nuevo acto lesivo se configura a partir de la comparación propia a determinar entre ambos actos agresores. (Figueroa, 2013).

Esto:

Sucederá cuando la sentencia final que ha identificado un acto como agresor de un derecho fundamental y ha declarado fundada la demanda, haya sido emitida en contravención al derecho constitucional vigente. En este supuesto, la represión del acto agresor de un derecho fundamental se formula sobre una resolución ejecutoriada que no ha alcanzado la calidad de cosa juzgada constitucional. (Castillo, 2009, p. 13)

Si existe identidad de agresión, el juez estimará el pedido de la parte accionante. A su turno, si no existe el requerimiento de identidad, será necesario desestimar el pedido y la parte afectada quedará en libertad de recurrir a la vía pertinente si considera que se ha consumado una agresión.

Se nota que para la agresión se determina con la potestad del magistrado de ir a tutelar el derecho, con el fin de no iniciar un nuevo proceso. Siempre que exista una manifiesta identidad de los actos que originaron la agresión. 


\section{b) Cumplimiento de lo ordenado en la sen- tencia de condena}

Para esta exigencia es fundamental distinguir que la represión de actos homogéneos no se relaciona con los aspectos fijados por el artículo $22^{\circ}$ del Código Procesal Constitucional.

En efecto, si existe un mandato firme en sede constitucional y éste no es acatado por la parte obligada, es entonces necesario recurrir a los apercibimientos de multas compulsivas, acumulativas e inclusive destitución, mas no será exigible recurrir a la figura de represión de actos homogéneos.

Esto se debe a la necesaria diferenciación de medida sustantivamente alterna de esta institución frente a los apercibimientos en sede constitucional y no a un carácter de complementariedad de la figura de represión de actos homogéneos.

En ese sentido, es vital establecer que esta institución tenga una exigencia diferenciada de las demás instituciones procesales constitucionales, en tanto existe un nuevo acto agresor que reviste características muy similares a aquella agresión que dio lugar a que se estimara la demanda. (Figueroa, 2013)

Esta institución tiene una particularidad. Para su configuración, se requiere una nueva agresión al mismo derecho fundamental reconocido en un previo proceso, mediante hechos idénticos que sustentan la emisión de la sentencia ejecutoriada.

La represión de actos homogéneos no es un mecanismo para asegurar el cumplimiento de una sentencia, sino un mecanismo para atacar actos futuros sustancialmente homogéneos al acto agresor. Si emitida una sentencia en la que se declara que determinado acto agrede un derecho fundamental y ordena la cesación del mismo, y el demandado no cumple con lo ordenado, lo que procede no es la represión de actos homogéneos, sino los apremios procesales correspondientes para hacer cumplir el fallo, apremios como por ejemplo los recogidos en el artículo $22^{\circ} \mathrm{CP}$ Constitucional. (Castillo, 2009, p. 12)

La represión de actos lesivos homogéneos es un mecanismo que tiene por objeto hacer frente a actos futuros, similares al acto agresor. En el contexto de un precedente resolutivo en donde se pronuncia declarando que determinado acto vulnera un derecho fundamental y de manera conjunta ordena el cese del anterior, sin embargo, el demandado no cumple con lo escrito en la resolución no corresponderá como pretensión acudir a esta institución sino en vez de ella a los apremios procesales que corresponde hacer cumplir con el fallo.

\section{ELEMENTOS DE LA REPRESIÓN DE ACTOS LESIVOS HOMOGÉNEOS}

\subsection{Elementos Subjetivos}

Se distingue dos aspectos: las características de la persona afectada por el acto homogéneo y las características de la fuente u origen del acto. (Exp. 01495-2012-PA/TC, 2012)

\section{Características de la persona}

Según la sentencia del TC (EXP. N. ${ }^{\circ} 04878$ 2008-PA/TC), citando al jurista Eduardo Ferrer Mac-Gregor, la represión de actos lesivos homogéneos puede ser invocada por:

- La persona quien fue lesionada por los hechos de la primera afectación que se resolvió bajo sentencia.

- Por cualquier persona en el caso de los derechos difusos.

- Por cualquier integrante del grupo en el caso de derechos colectivos.

- Por cualquier persona que se encuentre en una situación igual a la considerada como un estado de cosas inconstitucional, en el caso de los derechos individuales homogéneos.

Este último supuesto, es el que importa para el caso materia de análisis, dado que todos 
los docentes que laboraron bajo el régimen de la Ley del Profesorado N. ${ }^{\circ} 24029$ y su modificatoria $\mathrm{N}^{\circ} 25212$, han sido vulnerados en el derecho fundamental de la remuneración, según el inciso 20 del art. 53 del Código Procesal Constitucional.

\section{Características de la fuente}

La fuente del acto lesivo debe corresponder al mismo sujeto agresor o a la persona jurídica que a través de cualquiera de sus funcionarios, hubiere sido el primer funcionario agresor $u$ otro, consolidándose una nueva agresión a la parte afectada y determinándose la necesidad de una tutela vía la represión de actos lesivos homogéneos. (Figueroa, 2013)

Se denomina a esta exigencia como la unidad que determinara la fuente siempre que fuesen determinados quienes cometieron la agresión, quien vuelve a cometerla respecto del acto denominado homogéneo.

\subsection{Elementos objetivos}

Los elementos objetivos están orientados a confrontar que el nuevo acto que requiere tutela jurídica y el acto declarado lesivo de derechos fundamentales por el órgano jurisdiccional, compartan los mismos hechos o sustancialmente contengan elementos fácticos similares y también corresponde evaluar que el nuevo acto tenga una relevancia constitucional, vale decir, que afecte un determinado derecho constitucional.

Se debe de tener en cuenta:

Que el acto sobrevenido deberá ser considerado como homogéneo aún si trae consigo otras circunstancias fácticas no presentes en el acto declarado inconstitucional, siempre que tales circunstancias sean irrelevantes para considerar configurada la agresión constitucional (por lo que no debe afectar a la esencia o sustancia del acto). (Castillo, 2009, p. 14)
Los hechos que no gocen de relevancia para el proceso y que no se presenten de manera directa en el acto que vulnera algún tipo de derecho fundamental, no afectara en la naturaleza de la declararon de la institución de la represión de actos lesivos homogéneos.

\section{Homogeneidad del nuevo acto respec- to a uno anterior}

Regulado por el Código Procesal Constitucional, en su artículo $60^{\circ}$, que manifiesta que el nuevo acto agresor debe representar características similares respecto a aquel primer acto que constituyó la agresión declarada contraria a la Constitución.

Tienen que ser idénticas las razones en medida que si no pertenecen al mismo ámbito no figuraría dentro de los alcances de la represión de actos lesivos homogéneos.

En vía de ejemplo, si un trabajador fue despedido inconstitucionalmente a través de un despido nulo y logra, como es de preverse, su reposición, sería objeto de tutela en una petición de represión de actos homogéneos, nuevamente otro despido nulo. A su vez, si el nuevo despido se basara en un despido fraudulento, sustantivamente distinto al acto fuente, consideramos que es de inferir que ya no nos encontramos frente a un acto a ser reprimido vía esta institución procesal. (Figueroa, 2013)

Cuando los hechos facticos realmente infieren en la naturaleza de la institución procesal de la represión de actos lesivos homogéneos, impide al demandante poder acudir a esta institución los que de manera clara, se denota que no todos los procesos pueden ser resueltos por medio de esta intuición, sino que es necesario que el operador del derecho identifique realmente cuales son los casos que se pueden resolver gracias a este mecanismo constitucional así como identifique que casos no se desarrollan por el mismo. 


\section{Manifiesta homogeneidad}

La experiencia judicial en estos avatares nos conduce a afirmar que en rigor ésta es la exigencia más compleja a propósito de la acreditación de los actos homogéneos con relación al acto agresor, en tanto debe existir un necesario criterio de conexión entre los actos sujetos a comparación, en los cuales bien pueden coincidir sujeto, fuente y características similares en la forma del acto, pero no necesariamente coinciden respecto a la exigencia de manifiesta homogeneidad pues en este ítem concreto cuanto se exige es que haya características evidentemente similares con relación al acto fuente agresor, lo cual, las más de las veces, no se cumple cabalmente por parte de quienes resultan solicitantes respecto de una represión de actos homogéneos. (Figueroa, 2013)

Si no hay homogeneidad, se tendrá que rechazar la solicitud y empezar un nuevo proceso en otra vía, es por esa razón que sea una exigencia mayor a comparación de las otras exigencias.

\section{TUTELA JURISDICCIONAL EFECTIVA}

En la actualidad, en todo estado Constitucional de Derecho, para poder dar solución a un conflicto de intereses no podemos hacer «justicia por mano propia», siendo la autocomposición y la heterocomposición mecanismos de solución de conflictos.

Hoy en día el estado promueve la autocomposición reservando en última instancia recurrir al órgano jurisdiccional, todo ello en pro de mantener la paz social, por lo que es de necesidad que el ente estatal sea capaz de crear instrumentos eficaces capaces de satisfacer las pretensiones de los justiciables, que son formulados ante el órgano jurisdiccional.

El derecho a la tutela jurisdiccional efectiva es aquel que permite el acceso a los órganos jurisdiccionales, pero como integrante de una sociedad, en donde el estado debe ofrecer las garantías necesarias y mínimas para que el proceso sea efectivo.

Por otra parte, De Bernardis (1985) define a la tutela jurisdiccional efectiva como:

La manifestación constitucional de un conjuntos de instituciones de origen eminentemente procesal, cuyo propósito consiste en cautelar en libre, real irrestricto acceso a todos los justiciables a la prestación jurisdiccional a cargo del Estado, a través de un debido proceso que revista os elementos necesarios, para hacer posible la eficacia del derecho contenido en las normas jurídicas vigentes o la creación de nuevas situaciones jurídicas, que culmine con la resolución final ajustada a derecho y con un contenido mínimo de justicia, susceptible de ser ejecutada coactivamente. (p. 27)

Nuestra Constitución (1993) en su artículo $139^{\circ}$, inciso 3 establece que:

Son principios y derechos de la función jurisdiccional: La observancia del debido proceso y la tutela jurisdiccional. Ninguna persona puede ser desviada de la jurisdicción predeterminada por la Ley, ni sometida a procedimiento distinto de los previamente establecidos, ni juzgada por órganos jurisdiccionales de excepción ni por comisiones especiales creadas al efecto, cualquiera sea su denominación. (Const., 1993, Art.139)

El artículo $4^{\circ}$ de nuestro Código Procesal Constitucional (2004):

Entiende por tutela procesal efectiva aquella situación jurídica de una persona en la que se respetan, de modo enunciativo, sus derechos de libre acceso al órgano jurisdiccional, a probar, de defensa, al contradictorio e igualdad sustancial en el proceso, a no ser desviado de la jurisdicción predeterminada ni sometido a procedimientos distintos de los previstos por la ley, a la obtención de una resolución fundada en derecho, a acceder a los medios impugnatorios regulados, a la imposibilidad de revivir procesos fenecidos, a la actuación 
adecuada y temporalmente oportuna de las resoluciones judiciales y a la observancia del principio de legalidad procesal penal. (Código Procesal Constitucional, 2004)

Entonces una vez más se reconoce la importancia de la tutela jurisdiccional efectiva, tanto en la Constitución como en el Código Procesal Constitucional definiéndola como presupuesto de acceso a la justicia, sin la que será imposible ser oído por el órgano jurisdiccional competente, además de las demás garantías que significa.

\section{LA REPRESIÓN DE ACTOS LESIVOS HO- MOGÉNEOS Y LA TUTELA JURISDICCIO- NAL EFECTIVA DEL PERSONAL DOCENTE QUE LABORÓ DURANTE LA VIGENCIA DE LA LEY DEL PROFESORADO N. ${ }^{\circ} 24029$ Y SU MODIFICATORIA LEY N. ${ }^{\circ} 25212$}

El objetivo general de esta investigación es describir la institución jurídica de la represión de actos lesivos homogéneos y la forma en que garantiza la tutela jurisdiccional efectiva al personal docente que laboró durante la vigencia de la Ley del Profesorado N. 24029 y su modificatoria Ley $\mathrm{N} .^{\circ} 25212$. Es así que, si bien el derecho peruano, a través de su legislación específicamente el Código Procesal Constitucional reúne una serie de instituciones que protegen derechos de valor constitucional. No obstante de la misma manera permite el resguardo del respeto al plazo razonable, ya que ante la existencia de una carga procesal excesiva debe de tenerse en cuenta de que la razón de este mecanismo constitucional es asegurar que las sentencias ejecutoriadas se cumplan de manera obligatoria y evitar nuevos procesos constitucionales cuyos hechos son similares y/o idénticos a aquellos que previamente han sido discutidos y calificados como lesivos de derechos fundamentales por lo que evitaría que el operador del derecho revisara nuevamente procesos con sentencias ejecutoriadas anteriormente, garantizándose de manera rápida y oportuna la tutela jurisdiccional efectiva. Mu- chas veces erróneamente se cree que esta institución de derecho constitucional es garantiza de sobremanera a la parte demandada frente al demandado, en un acto de carácter homogéneo por lo que una parte de la doctrina lo ha tildado de inconstitucional en el amparo, sin considerar la funcionalidad y mecánica del principio de cosa juzgada, así como los artículos $22^{\circ}$ y $59^{\circ}$ del Código Procesal Constitucional.

Uno de los mayores problemas en la administración de la justicia, se relaciona con la mora de procesos con respecto al plazo razonable de los procesos, lo cual se justifica por los operadores del derecho que esta situación se debe a que existe una excesiva carga procesal. Se ha constatado que por no aplicarse la Institución de represión de actos lesivos homogéneos, los procesos tramitados para conseguir el reconocimiento de la bonificación especial calculada en base al $30 \%$ de la remuneración total, amparado por el Art. $48^{\circ}$ de la Ley del Profesorado, tardan hasta, un poco más de cinco años, esto debido que el Poder Judicial enfrenta problemas de gestión por contar con un reducido presupuesto otorgado por parte del Ejecutivo lo que se materializa en que la excesiva carga procesal a la que se hace referencia, solamente lo soportan los juzgados laborales públicos y juzgados mixtos, que conocen demandas contenciosas administrativas provenientes de servidores docentes del sector educación.

En la presente investigación se ha propuesto la necesidad de aplicar la institución de los actos lesivos homogéneos para los procesos seguidos por el personal docente en el amparo de su derecho reconocido por la ley N. 24029 y su modificatoria Ley N. ${ }^{\circ} 25212$, lo que en realidad asegura el carácter urgente de los procesos constitucionales, lo que permitiera de una parte la no interposición de un nuevo proceso constitucional frente a la agresión o vulneración de un proceso en la etapa de ejecución, asimismo y más importante implica la actuación célere y oportuna frente a vulneraciones de los mismos derechos antes discutidos en un resolución previa. 
De los antecedentes internacionales y nacionales que sirvieron de cimiento a esta investigación, si bien se viene discutiendo si es pertinente la aplicación de esta institución procesal ya reconocida por ley, especialmente para este tipo de casos siendo más pertinente este mecanismo constitucional que recurrir a la vía contenciosa administrativa, que para otro tipo de procesos laborales es más viable, sin embargo este enfoque materia de estudio es diferente en cada uno de los casos, sin embargo la propuesta desarrollada en la presente, tiene una tendencia innovadora por lo que tiene un debate muy escaso.

Dentro de la presente investigación, la validación y confidencialidad de instrumentos realizados, principalmente el poder judicial, una de la principales limitaciones que tienen los operadores del derecho es la aglutinante carga procesal, sin embargo de tramitarse los procesos de reconocimiento de derechos y beneficios laborales del docente que laboró durante la vigencia de la Ley del Profesorado N. ${ }^{\circ} 24029$ y su modificatoria Ley $N .^{\circ} 25212$, si se respetaría el plazo razonable llegándose a resolver el proceso en un máximo de 1 año y 7 meses aproximadamente, claro que esta situación puede variar de acuerdo a la naturaleza del derecho reclamado y a la complejidad del proceso, así como a lo desavienes y situaciones específicas del poder judicial, sin embargo comparando ambos resultados podemos distinguir que la institución de represión de actos lesivos homogéneos hace frente a la problemática de la carga procesal, convirtiéndose en una respuesta grata para el personal docente.

Por lo que, se propone la necesaria aplicación de la institución de los actos lesivos homogéneos para los procesos seguidos por el personal docente en el amparo de su derecho reconocido por la ley $\mathrm{N} .^{\circ} 24029$ y su modificatoria Ley $\mathrm{N} .^{\circ} 25212$, lo que en realidad asegura el carácter urgente de los procesos constitucionales, lo que permitirá de una parte, la no interposición de un nuevo proceso constitucional fren- te a la agresión o vulneración de un proceso en la etapa de ejecución; asimismo y más importante, implica la actuación célere y oportuna frente a vulneraciones de los mismos derechos antes discutidos en una resolución previa.

\section{CONCLUSIONES}

Primera.- El instrumento de la represión de actos lesivos homogéneos para el trámite de los procesos seguidos por el personal docente al amparo de sus derechos reconocidos por la ley $\mathrm{N} .{ }^{\circ} 24029$ y su modificatoria Ley $\mathrm{N} .^{\circ}$ 25212 , servirá para hacer más eficaces los procesos. Es decir, a través de este instrumento se reducirían los plazos significativamente respecto al que actualmente se emplea para obtener una sentencia favorable, que aproximadamente es de nueve meses, además garantizará su pronta ejecución en razón a que, en un procedimiento de represión de actos lesivos homogéneos, es posible apelar una resolución, pero sin efecto suspensivo; a diferencia del proceso contencioso administrativo que tiene una duración que puede llegar hasta los cinco años.

Segunda.- Es necesaria la introducción de reformas en los procesos de la administración pública, eso significa hacer uso de instrumentos más efectivos que los que poseemos, es en esa línea, que la represión de actos lesivos homogéneos significa una excelente opción para agilizar procesos que tienen correspondencia entre sí, después de un precedente vinculante. La Ley del profesorado N. ${ }^{\circ} 24029$ reconoce el derecho de percibir la bonificación especial, el cual viene a ser el 30\% del integro de la remuneración, que es reconocido a todos los docentes, este derecho puede ser atendido de mejor forma con el uso de la represión de actos lesivos homogéneos, lo cual aligeraría la carga en la administración pública, así como sería posible habituarse a procesos más céleres.

Tercera.- Con relación a la administración de justicia, si se logrará difundir el uso del instrumento de la represión de actos lesivos 
homogéneos, se garantizaría el respeto al principio de economía procesal, reduciendo además el desgaste de la actividad jurisdiccional como consecuencia de la proliferación de los procesos contenciosos administrativos.

La represión actos lesivos homogéneos de todas maneras garantiza la observancia del plazo razonable, ya que ante la existencia de una carga procesal excesiva debe de tenerse en cuenta de que la finalidad de este mecanismo constitucional eses asegurar que las sentencias ejecutoriadas se cumplan de manera obligatoria y evitar nuevos procesos constitucionales cuyos hechos son similares i/o idénticos a aquellos que previamente han sido discutidos y calificados como lesivos de derechos fundamentales, por lo que evitaría que el operador del derecho revisara nuevamente procesos con sentencias ejecutoriadas anteriormente, y como consecuencia inequívoca se aliviaría la carga procesal, entonces los operadores de justicia podrían ocuparse de otros casos que pudieron quedar relegados.

Cuarta.- La represión actos lesivos homogéneos realmente garantiza el respeto al plazo razonable, lo cual resultará en la creación de un espacio correcto para la realización de las demás garantías que constituyen la tutela jurisdiccional efectiva, como son el acceso a la justicia, debido proceso y la ejecución de sentencias. Entonces la represión de actos lesivos homogéneos supone el instrumento más conveniente para que el personal docente que laboro bajo el régimen de las leyes 24029 y 25012 peticione sus derechos, conforme a una tutela jurisdiccional efectiva, resumiéndose una vez más, que la justicia, en muchos casos, se plasma en cuestión de tiempo.

\section{REFERENCIAS}

De Bernardis, L. (1985). El derecho a la Tutela Jurisdiccional Efectiva. Lima: Cultural Cusco S.A.

Castillo. (2009). Análisis a la doctrina de la represión de actos homogéneos. Jurisprudencia de observancia obligatoria para abogados y jueces. Piura: Gaceta Constitucional.

Eto. (2016). La transversalización del derecho constitucional. http://repositorio.amag.edu. pe/bitstream/handle/123456789/690/MATERIAL\%20TRATADO\%20TRAVERSALIZACION.pdf? sequence $=4 \&$ isAllowed $=y$

Figueroa. (2013). Represion de actos homogéneos. https://edwinfigueroag.wordpress.com/ x-represion-de-actos-homogeneos/

Gonzales. (1985). El Derecho a la Tutela Jurisdiccional Efectiva. España: Editorias Civitas.

Rioja. (2012). El Proceso de Amparo Peruano. Lima: Jurista.

\section{REFERENCIAS JURÍDICAS}

Código Procesal Constitucional (31 de mayo de 2004). https://www.tc.gob.pe/tc/private/adjuntos/transparencia/pdf/marco_legal/Codigo_Procesal.pdf

Constitución Política del Perú [Const.] (29 de diciembre de 1993). https://www.minjus.gob. pe/wp-content/uploads/2019/05/Constitucion-Politica-del-Peru-marzo-2019_WEB.pdf

Convención Americana sobre Derechos Humanos (22 de noviembre de 1969). https:// www.corteidh.or.cr/tablas/17229a.pdf

Decreto Supremo [N. ${ }^{\circ}$ 051-91- PCM]. (04 de marzo 1991). http://files.servir.gob.pe/WWW/files/ normas\%20legales/DS\%20051-91-PCM.pdf

Ley del Profesorado. [Ley N. ${ }^{\circ}$ 24029] (14 de diciembre de 1984). http://www.minedu.gob. pe/normatividad/leyes/Ley24029.php

Modifican la Ley del Profesorado. [Ley N. ${ }^{\circ}$ 25212] (20 de mayo de 1990). http://www4.congreso. gob.pe/historico/cip/materiales/profesorado/ Ley_25212_Prorroga_ley_profesorado.pdf

Sentencia N.o 04878-2008-PA/TC. (2009). https:// tc.gob.pe/jurisprudencia/2009/04878-2008AA\%20Resolucion.pdf 
Sentencia N.o 01495-2012-PA/TC (2012). https:// www.tc.gob.pe/jurisprudencia/2013/014952012-AA.html

Sentencia N. ${ }^{\circ}$ 01345-2013-PA/TC (2013). https:// www.tc.gob.pe/jurisprudencia/2014/013452013-AA.html

Texto Único Ordenado del Código Procesal Civil (23 de abril de 1993). https://iberred.org/ sites/default/files/codigo-procesal-civilper.pdf

Texto Único Ordenado de la Ley Orgánica del Poder Judicial (02 de junio de 1993). https://www.minjus.gob.pe/wp-content/uploads/2014/09/DGDOJ-LeyOrg\%C3\%A1 nica-Poder-Judicial-y-Ministerio-P\%C3\%BAblico.pdf 